\title{
Target-Dependent Regulation of Neurotransmitter Specification and Embryonic Neuronal Calcium Spike Activity
}

\author{
Qian Xiao, Lin Xu, and Nicholas C. Spitzer \\ Neurobiology Section, Division of Biological Sciences, Kavli Institute for Brain and Mind, University of California, San Diego, La Jolla, California $92093-0357$
}

\begin{abstract}
Neurotransmitter specification has been shown to depend on genetic programs and electrical activity; however, target-dependent regulation also plays important roles in neuronal development. We have investigated the impact of muscle targets on transmitter specification in Xenopus spinal neurons using a neuron-muscle coculture system. We find that neuron-muscle contact reduces the number of neurons expressing the noncholinergic transmitters GABA, glycine, and glutamate, while having no effect on the incidence of ChAT expression. We show that muscle activity is necessary for target-dependent reduction of noncholinergic transmitter expression. In addition, we demonstrate that coculture with muscle cells suppresses early spontaneous calcium spike activity in neurons and the presence of muscle cells abolishes activity-dependent transmitter specification. The results indicate that target-dependent regulation can be crucial in establishing neurotransmitter phenotypes and altering early neuronal excitability.
\end{abstract}

\section{Introduction}

Neurotransmitters provide the fundamental basis for neurons to communicate with their targets, and the expression of proper transmitters is critical for normal functioning of the nervous system. Developmental specification of transmitter phenotype is a complex process that involves the interplay of several different mechanisms and pathways. Selective gene expression specifies neuronal identities (Lee and Pfaff, 2001). Misexpression of the homeobox gene MNR2 in chick embryos induces ectopic expression of choline acetyltransferase (ChAT) (Tanabe et al., 1998). LMO4 induces GABAergic differentiation in V2b interneurons and blocks Lhx3-induced glutamatergic differentiation in V2a interneurons in mouse spinal cord (Joshi et al., 2009). Early neuronal activity also plays important roles in transmitter specification (Spitzer, 2006). Spontaneous brief elevations of intracellular calcium (calcium spikes) are observed in embryonic Xenopus spinal neurons both in culture and in vivo ( $\mathrm{Gu}$ et al., 1994). Different classes of neurons express different patterns of calcium spikes, and the frequency of calcium spikes specifies transmitter expression in a homeostatic manner (Borodinsky et al., 2004). Activity is required for expression of tyrosine hydroxylase during development of dopaminergic rat sensory ganglion neurons (Brosenitsch and Katz, 2002) and activation of NMDA receptors specifies the glutamatergic phenotype in cultured rat hypothalamic neurons (Liu et al., 2008).

Received Nov. 15, 2009; revised March 10, 2010; accepted March 16, 2010.

This research was supported by National Institutes of Health Grant R01NS15918 to N.C.S. We thank members of our laboratory for helpful discussions and technical assistance, Darwin Berg, Davide Dulcis, Kurt Marek, and Xavier Nicol for comments on this manuscript, and A. de la Torre and I. Hsieh for technical support.

Correspondence should be addressed to either Qian Xiao or Nicholas C. Spitzer, Neurobiology Section, Division of Biological Sciences, University of California, San Diego, 9500 Gilman Drive, La Jolla, CA 92093-0357, E-mail: qxiao@ ucsd.edu or nspitzer@ucsd.edu.

DOI:10.1523/JNEUROSCI.5659-09.2010

Copyright $\odot 2010$ the authors $\quad 0270-6474 / 10 / 305792-10 \$ 15.00 / 0$
At embryonic stages, developmentally regulated extrinsic factors determine neural fate in combination with factors intrinsic to the neurons. Target-derived factors regulate axonal and dendritic growth, synapse formation and neuronal survival (Zweifel et al., 2005). Neurotrophic factors can drive acetylcholine expression instead of noradrenaline in mammalian sympathetic ganglion neurons (Furshpan et al., 1976; Landis and Keefe, 1983; Nawa and Patterson, 1990; Francis and Landis, 1999). Neurotrophin transcript expression peaks early in embryonic development and declines at later times (Timmusk et al., 1993), consistent with developmental roles of these factors. Muscle tissue is an important source for many trophic factors including NT-3/4/5, BDNF, NGF, GDNF, CNTF, and LIF (Timmusk et al., 1993; Funakoshi et al., 1995; Fernandes et al., 1998; Fu et al., 1998; Francis and Landis, 1999). Muscle-derived factors are critical for neuronal expression of ChAT (Lams et al., 1988) and acetylcholinesterase (AChE) (Jiang et al., 2003). Eliminating these factors by axotomy or muscle removal often results in cell death or loss of ChAT and AChE expression. The loss can be rescued by application of trophic factors, including NT-4/5, BDNF, and GDNF (Kou et al., 1995; Fernandes et al., 1998). Muscle-derived factors are also critical for establishing mature properties of synapses (Liou and Fu, 1997; Nick and Ribera, 2000). These studies demonstrate the importance of target-derived factors in neuronal development. However, the interplay between these factors and early neuronal activity, as well as the mechanisms associated with this muscle-dependent regulation of neuronal development, are yet to be understood.

Here we report that cultured muscle cells suppress noncholinergic transmitter expression in Xenopus spinal cord neurons that grow and make contact with them. We show that retrogradely transported signals and muscle activity are key players in targetdependent transmitter specification. Muscle cells also suppress early calcium spike activity in contacted neurons and abolish 
A

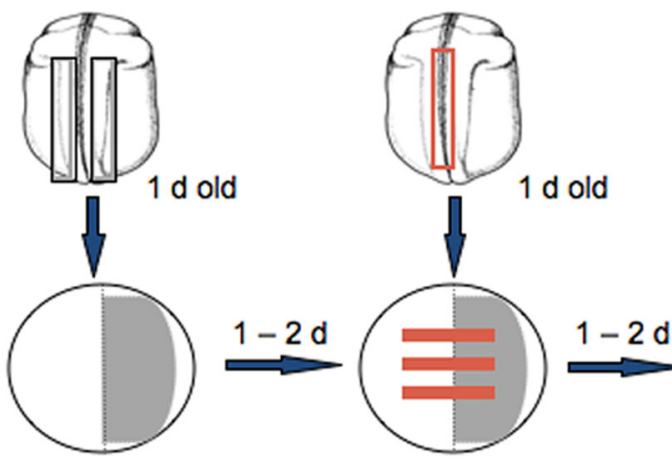

B

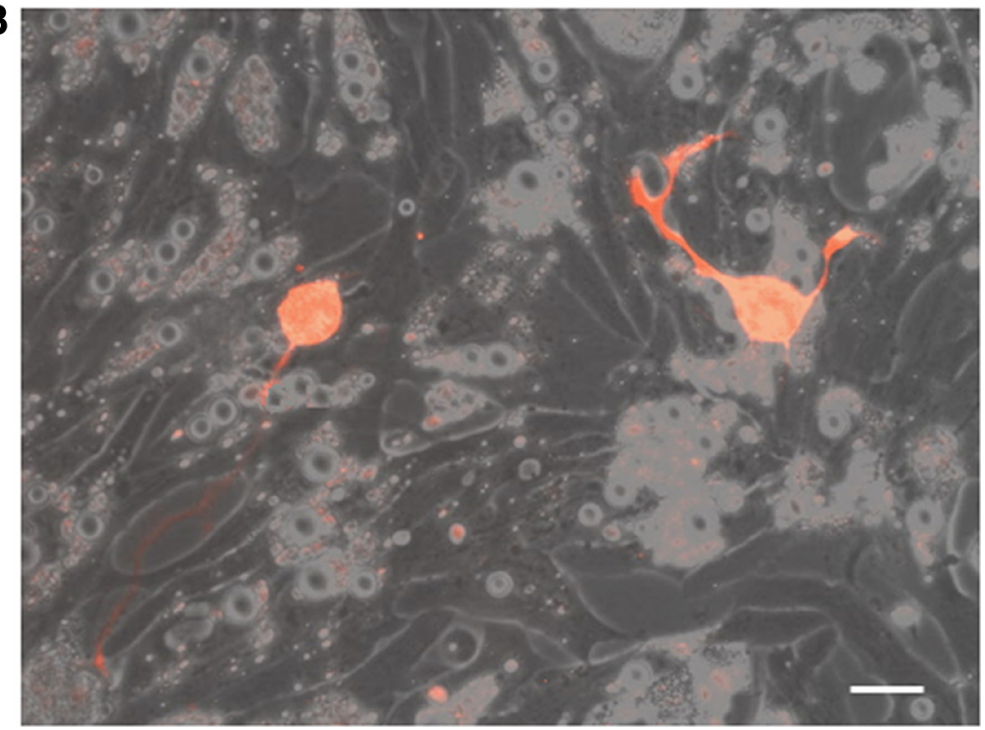

Figure 1. Neuron-muscle coculture system. $\boldsymbol{A}$, Diagram illustrating the procedure for establishing neuron-muscle cocultures Areas in which muscle cells are plated are shown in gray and areas in which neurons are located are shown in red. $\boldsymbol{B}$, Neurons growing on muscle cells. Neurons were dissociated from rhodamine dextran-injected embryos. Scale bar, $10 \mu \mathrm{m}$.

were then plated. For the control condition, $3 \mathrm{ml}$ of culture medium were incubated in a culture dish without muscle cells and harvested after $48 \mathrm{~h}$. Neurons were allowed to grow in each medium for $24 \mathrm{~h}$ before they were fixed and stained.

Immunocytochemistry. Cultures were fixed in $4 \%$ paraformaldehyde, 0.1 or $0.025 \%$ glutaraldehyde, PBS, pH 7.4, for $10-15 \mathrm{~min}$ at $20^{\circ} \mathrm{C}$. Preparations were washed in PBS for $30 \mathrm{~min}$, incubated in a blocking solution of $1 \%$ fish gelatin (Sigma) for $0.5 \mathrm{~h}$ at $20^{\circ} \mathrm{C}$, and stained overnight at $4^{\circ} \mathrm{C}$ using the following antibodies: mouse IgG anti- $\beta$-tubulin (Sigma), 1:1000; guinea pig anti-GABA (Millipore Bioscience Research Reagents), 1:1000; rabbit anti-glycine (Millipore Bioscience Research Reagents), 1:50; rabbit anti-glutamate (Sigma), 1:10,000; mouse IgM anti-HNK-1 (Sigma), 1:100; rabbit anti-GAD (Enzo Lifescience), 1:200; and rabbit anti-VGLUT-1 (Sigma), 1:5000. Fluorescent secondary antibodies (Invitrogen) were used at $1: 300$ for $1-2 \mathrm{~h}$ at $20^{\circ} \mathrm{C}$. Immunoreactivity was examined on a Zeiss Axioskop with a $40 \times$ water objective using a xenon arc lamp attenuated by neutral density filters, and the appropriate excitation and emission filters for Alexa 488 and Alexa 594 fluorophores. Images were acquired and analyzed with Axiovision (Zeiss). Neurons were considered immunopositive when the average pixel intensity of the neuronal cell body was 10 times higher than that of the background tissue culture plastic dish. The average pixel intensity of immunonegative neurons was $<5$ times that of background.

In situ hybridization. A $1.5 \mathrm{~kb}$ probe cDNA template for ChAT was a generous gift from Dr. Margaret Saha (College of William and Mary, Williamsburg, VA). It was cloned in a PCR4 vector and antisense RNA probe was transcribed using the Megascript Kit (Ambion)

activity-dependent transmitter specification without neuronal contact.

\section{Materials and Methods}

Cell culture. Mixed cell cultures containing neurons and muscle cells were prepared from Xenopus laevis neural tube (stage 20) embryos (Ribera and Spitzer, 1989; Holliday and Spitzer, 1990). Myotomes and spinal neural tube were dissected from embryos using jewelers' forceps and tungsten needles in the presence of $1 \mathrm{mg} / \mathrm{ml}$ collagenase (Boehringer Mannheim). Cells were dissociated for $45 \mathrm{~min}$ in calcium-magnesium-free medium containing the following (in $\mathrm{mm}$ ): $58.8 \mathrm{NaCl}, 0.67 \mathrm{KCl}, 0.4$ EDTA, 4.6 Tris, $\mathrm{pH}$ adjusted to 7.8 with $\mathrm{HCl}$. Dissociated muscle cells from a single embryo were plated on one side of a $35 \mathrm{~mm}$ tissue plastic culture dish, either as a dense lawn or in a striped fashion. Neurons from a single embryo were plated in stripes across the whole dish, 0 or $24 \mathrm{~h}$ after plating muscle cells unless otherwise specified. Neuron-alone cultures were prepared as previously described (Gu and Spitzer, 1995; Borodinsky et al., 2004). Cultures were allowed to grow $24 \mathrm{~h}$ after plating neurons before they were fixed and stained (Fig. $1 A, B$ ). Culture medium contained the following (in mM): $116.6 \mathrm{NaCl}, 0.67 \mathrm{KCl}, 1.31 \mathrm{MgSO}_{4}, 2 \mathrm{CaCl}_{2}$ and 4.6 Tris, $\mathrm{pH}$ adjusted to 7.8 with $\mathrm{HCl}$. There are $\sim 1500$ neurons in the spinal neural tube at these stages (Hartenstein, 1989) and only those cultures with $>100$ neurons were scored (Holliday and Spitzer, 1993).

Conditioned medium. Myotomes from six neural tube stage embryos were dissected, dissociated, plated, and allowed to grow for $48 \mathrm{~h}$ in $3 \mathrm{ml}$ of culture medium. In some experiments, neurons from six neural tube stage embryos were dissociated and plated $24 \mathrm{~h}$ following muscle plating. Conditioned medium $(2.5 \mathrm{ml})$ was harvested from each dish and transferred to a new culture dish, in which neurons from a single neural tube stage embryo in the presence of digoxigenin-labeled UTP (Roche). Cell cultures were fixed in $4 \%$ paraformaldehyde for $1 \mathrm{~h}$, washed for $30 \mathrm{~min}$ in PBS, and treated with $0.2 \mathrm{M} \mathrm{HCl}$ for $10 \mathrm{~min}$. Hybridization was performed at $60^{\circ} \mathrm{C}$ overnight with a probe concentration of $40 \mathrm{ng} / \mathrm{ml}$. Preparations were washed in $0.2 \times$ saline sodium citrate buffer for $1 \mathrm{~h}$ at $60^{\circ} \mathrm{C}$ and $30 \mathrm{~min}$ at $20^{\circ} \mathrm{C}$ before staining overnight at $4^{\circ} \mathrm{C}$ using anti-digoxigenin antibody (coupled to alkaline phosphatase) diluted to a final concentration of 1:1000 in $10 \%$ goat serum. Cultures were then rinsed five times in $0.5 \%$ Triton $\mathrm{X}-100$ in PBS and two times in alkaline phosphatase buffer at $20^{\circ} \mathrm{C}$ and incubated in BM Purple (Roche) for 12-36 h for signal development. Cultures were washed in PBS for $1 \mathrm{~h}$ before anti- $\beta$-tubulin antibody was introduced following the protocol described above, to help visualize neurons.

Imaging. Fluorescence of the calcium indicator Fluo-4 AM (Invitrogen) was used to study elevations of $[\mathrm{Ca}]_{\mathrm{i}}$ in neurons. Cultured cells were incubated for $45 \mathrm{~min}$ to $1 \mathrm{~h}$ in culture medium containing $5 \mu \mathrm{M}$ indicator and $0.01 \%$ Pluronic F-127 detergent (Invitrogen) and washed in culture medium before imaging. Images were acquired at $0.2 \mathrm{~Hz}$ for $30 \mathrm{~min}$ periods with a Bio-Rad laser confocal system with a $20 \times$ waterimmersion objective. Image stacks were imported into NIH ImageJ for analysis. Calcium transients were initially identified by eye. For each neuron of interest, the cell body was traced using the line-drawing tools in the program and the average pixel intensity was determined. This measurement was repeated for every image in the stack and the values were exported to Microsoft Excel for analysis. These data were plotted against the time of image acquisition to yield fluorescence kinetics and intensity. Calcium transients were scored as spikes when the amplitude exceeded twice the baseline variation during the previous $10 \mathrm{~min}$ and the rise time was complete within $5 \mathrm{~s}$. Four or five cultures were imaged for each experiment and the incidence of spiking neurons was calculated by 
dividing the total number of neurons that spiked at least once during the $30 \mathrm{~min}$ imaging period by the total number of neurons imaged in that culture dish.

Pharmacology. Stock concentrations of drugs were $1 \mathrm{~mm}$ veratridine and $100 \mathrm{~mm}$ $\alpha$-bungarotoxin (BgTx). Veratridine and BgTx were added immediately before plating neurons, with final concentrations of $1 \mu \mathrm{M}$ and 250 $\mathrm{nM}$, respectively.

Channel overexpression. The hKir2.1 construct was a generous gift from Dr. Eduardo Marban (Johns Hopkins University, Baltimore, MD). The gene was subcloned into a Bluescript vector and mRNA was transcribed using the mMessage mMachine kit (Ambion). Capped mRNA [5-10 $\mathrm{nl}$ of a $0.01-0.1 \mathrm{mg} / \mathrm{ml} \mathrm{mRNA}$ solution in $10 \%$ Marc's Modified Ringer's solution (MMR) (100 $\mathrm{mm} \mathrm{NaCl}, 2 \mathrm{~mm} \mathrm{KCl}, 1 \mathrm{~mm} \mathrm{MgSO}_{4}, 5 \mathrm{~mm}$ HEPES, 0.1 mM EDTA, $2 \mathrm{~mm} \mathrm{CaCl}_{2}$, $\mathrm{pH}$ adjusted to 7.8$), 6 \%$ Ficoll] was coinjected with a cascade blue or rhodamine red $30 \mathrm{kDa}$ dextran $(30 \mathrm{mg} /$ $\mathrm{ml}$ ) into both blastomeres at the two-cell stage by using a picospritzer (Picospritzer III). Control injections consisted of fluorescent dextran alone (30 $\mathrm{mg} / \mathrm{ml}$ in 10\% MMR).

Statistical analysis. Means and SEs were calculated using Microsoft Excel. Statistical analyses were performed using online software provided by BrightStat (www.brightstat.com). For comparison among more than two groups, the nonparametric Kruskal-Wallis ANOVA test was used and followed by the nonparametric Conover post hoc test. For comparison between two groups, the MannWhitney $U$ test was used. Data are presented as mean \pm SEM. Results are considered significant when $p$ is $<0.05$.

\section{Results}

Neuron-muscle coculture reduces the number of neurons expressing noncholinergic neurotransmitters

To investigate the influence of muscle cells on neurotransmitter expression, we designed a coculture system in which muscle cells dissociated from a neural tube stage Xenopus embryo were plated in a lawn on one-half of a culture dish and spinal neurons dissociated from another neural tube stage embryo were plated in stripes across the whole dish 1 day later (Fig. $1 A, B$ ). This stage is before the formation of nervemuscle contacts in vivo (Kullberg et al., 1977). The procedure allowed neurons on the muscle side to form nerve-muscle contacts while neurons on the blank side grew free of contacts. Muscle contractions were observed on the neuron-muscle side $1 \mathrm{~d}$ after plating neurons, consistent with the formation of neuromuscular junctions (NMJs) during this period (Spitzer and Lamborghini, 1976). The incidence of functional NMJs in this coculture system is high (Henderson et al., 1984) and contractions were not observed when muscle cells were plated alone.

The numbers of neurons expressing noncholinergic neurotransmitters, GABA, glycine, and glutamate, were compared between the muscle side and the blank side within the same culture dish, by assaying GABA, glycine, and glutamate immunoreactivity at $1 \mathrm{~d}$ in vitro. Neuronal identity was recognized by both morphology and immunostaining for neuron-specific $\beta$-tubulin (Fig.
B
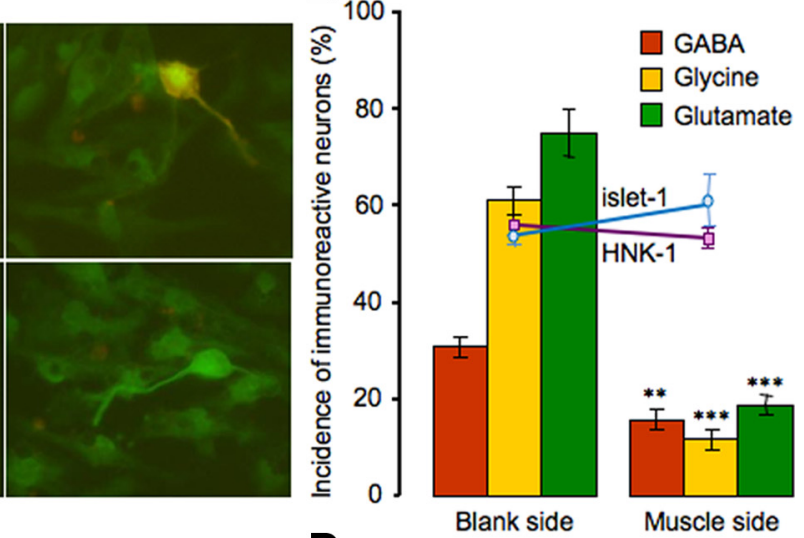

D

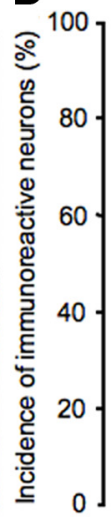

GAD

VGIUT 1

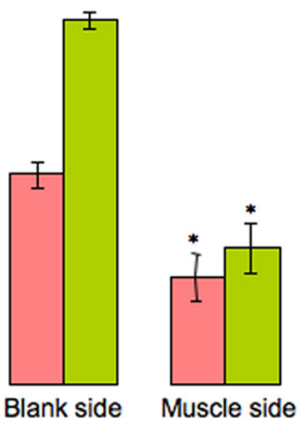

\section{Blank side Muscle side}

Figure 2. Muscle cells suppress noncholinergic neurotransmitter expression. $A$, Immunostaining of neurons for GABA (red) and Ute and the muscle side (right). Top, Positive staining for GAD; bottom, negative staining. D, GAD and VGluT1 expression is reduced on the muscle side ( ${ }^{*} p<0.05$, compared with the blank side; $n>5$ cultures per transmitter marker, $>100$ neurons per culture). Error bars indicate SEM. Scale as for $\boldsymbol{B}$. The Mann-Whitney $U$ test was used to determine statistical significance.

$2 A)$. Muscle cells pick up light $\beta$-tubulin staining both in vitro and in vivo (S. B. Sann, unpublished data). However, this does not affect recognition and counting of neurons because their morphology is distinct from that of muscle cells. Neurons expressing the three noncholinergic transmitters on the muscle side constitute a significantly smaller percentage of the total neurons scored when compared with the blank side (Fig. 2 B). Similar results were obtained when neurons were plated immediately after plating muscle cells. Approximately $10 \%$ of neurons grew axons long enough to make contacts with each other, both on the blank and the muscle side. However, scoring transmitter expression in neurons with and without contact with other neurons on either side did not reveal differences between these two groups. Because the culture medium does not contain any amino acids, if neurons release these compounds they may be unable to reload their transmitter pool. Accordingly we examined the effect of muscle cells on the expression of the transmitter-related proteins glutamic acid decarboxylase (GAD) and vesicular glutamate transporter 1 (VGLuT1). The results demonstrate decreased incidence of staining for GAD and VGluT1 on the muscle side of culture dishes, consistent with staining for GABA and glutamate (Fig. 2C,D). 


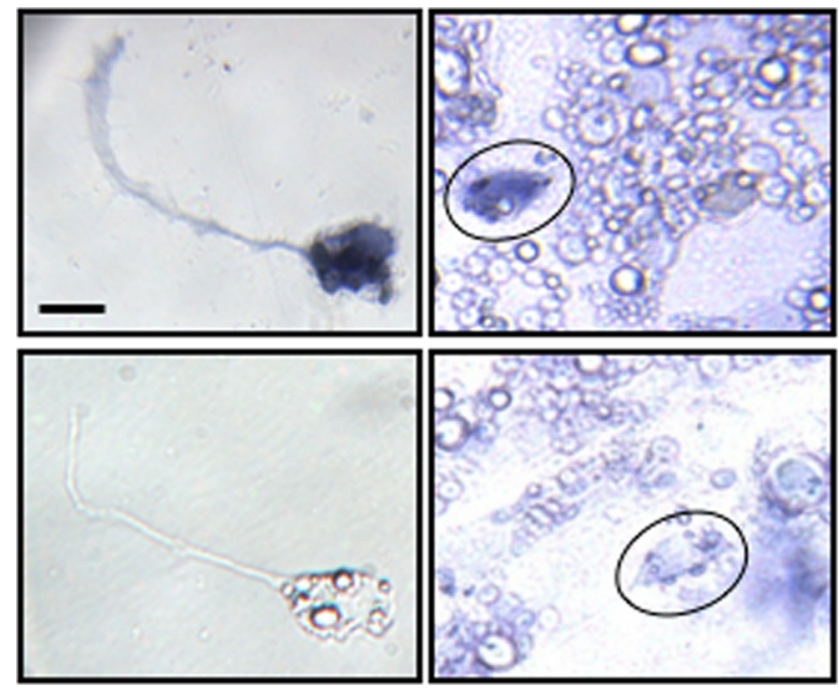

Figure 3. Neuronal ChAT expression is independent of muscle regulation. In situ hybridization for ChAT on the blank side (left) and the muscle side (right; neurons circled). Top, Positive staining; bottom, negative staining. Scale bar, $10 \mu \mathrm{m}$.
Thus, the presence of muscle cells reduces the fraction of neurons expressing noncholinergic transmitters only on the muscle side of the culture dish. The incidence of the three noncholinergic transmitters exceeds $100 \%$, suggesting coexpression. This result is in agreement with previous observations of transmitter coexpression in Xenopus spinal neurons at early stages of development, both in vitro and in vivo (Borodinsky et al., 2004; Root et al., 2008).

The reduction in number of neurons expressing noncholinergic transmitters could result from a decrease in differentiation or a lower survival rate of neurons that are genetically programmed to express noncholinergic phenotypes. However, the total number of neurons on each side was not significantly different $(109 \pm 10$ neurons on the blank side and $103 \pm 15$ neurons on the muscle side, $n=3$ cultures). We scored the number of neurons expressing HNK-1, an intrinsic molecular marker for a subtype of noncholinergic embryonic neurons (Somasekhar and Nordlander, 1997). There was no difference in the percentage of HNK-1-immunoreactive (-IR) neurons between the blank side $(55 \pm 1 \%)$ and the muscle side $(53 \pm 1 \%)$. Staining of islet- 1, a molecular marker for noncholinergic sensory neurons and motor neurons (Pfaff et al., 1996), also revealed no statistically significant difference in its expression in neurons on either side (52 \pm $1 \%$ on the blank side and $59 \pm 3 \%$ on the muscle side) (Fig. 2 B). These results indicate that cell death is unlikely to play a role in reducing the percentage of noncholinergic neurons. Data obtained at $6 \mathrm{~h}$ after plating demonstrated that culturing of neurons in the absence of muscle cells does not increase the proportion of the GABA-, glycine-, and glutamateimmunoreactive cells over the $24 \mathrm{~h}$ period. Thus, muscle cells do not exert their effects by preventing increases in proportions of aminoacidergic neurons.

To examine the impact of neuronmuscle coculture on cholinergic neurons, we used in situ hybridization to identify neurons expressing choline acetyltransferase (ChAT) (Fig. 3). Neuronal identity was recognized by morphology and fluorescent immunostaining for neuronspecific $\beta$-tubulin (data not shown). On both sides of the cultures, $\sim 30 \%$ of the neurons express ChAT $(28 \pm 2 \%$ on the blank side and $31 \pm 1 \%$ on the muscle side; not statistically different), suggesting that the number of cholinergic neurons is not affected by the presence of muscle cells. In situ signal for ChAT was observed in muscle cells as well, consistent with their release of ACh (Fu et al., 1998).

Figure 4. Cell contacts and not diffusible factors regulate muscle-dependent suppression of noncholinergic neurotransmitter expression. $A$, Schematic view of the lattice neuron-muscle coculture and groups of neurons with different interactions with muscles. $\boldsymbol{B}$, Neurons with or without cell body interaction with muscle show similar levels of reduction in incidence of noncholinergic transmitter expression, when compared with the no-contact group. There is no significant difference in noncholinergic transmitter expression between axon-M and soma-M groups $\left({ }^{* *} p<0.01\right.$, ${ }^{* * *} p<0.001 ; n>$ 5 cultures per condition per transmitter, $>100$ neurons per culture). $C$, The incidence of expression of the three noncholinergic transmitters in the no-contact group from $\boldsymbol{B}$ (GABA in red, glycine in yellow, and glutamate in green) does not change as a function of distance from neuronal soma to muscle cells ( $>100$ neurons scored at each distance for each transmitter). $\boldsymbol{D}$, The incidence of expression of the three noncholinergic transmitters does not differ between the control condition and conditioned medium condition (muscle alone or muscle cocultured with neurons) ( $n>5$ cultures per condition per transmitter, $>100$ neurons per culture). Error bars indicate SEM. The Kruskal-Wallis test and Conover post hoc test were used to determine statistical significance.

\section{Mechanism by which muscle cells induce suppression of noncholinergic neurotransmitter expression} Muscle cells could regulate neurotransmitter expression by several different means. On the muscle side, direct contact between neuronal somata and muscle cells allows physical interaction between membrane surface molecules, and axonmuscle contact permits retrograde trans- 
port of trophic factors that are normally taken up at the distal ends of axons (Zweifel et al., 2005). Diffusible factors released by muscle are present in the culture medium throughout the entire dish, with higher concentrations on the muscle side compared with the blank side. Extracellular matrix molecules are also released by the muscle and act locally on neurons growing in contact with muscle cells. To determine which of these interactions drives the reduction in expression of noncholinergic transmitters, we modified the coculture system to produce a lattice culture in which stripes of neurons are plated on stripes of muscle cells, instead of a muscle lawn (Fig. 4A). These cultures generate three classes of neurons: (1) those with no contact with muscle, (2) those with both axon and soma contacts with muscle (soma-M group), and (3) those with only axon contact with muscle (axon-M group).

The first class, growing on the blank side, may receive a low concentration of diffusible factors but their axons and somata remain free of muscle contact; thus other forms of interaction have little if any impact on them. The second class of neurons can receive retrograde signals and a higher concentration of diffusible factors. The neurons in this group can also interact with extracellular matrix and surface-bound molecules. The third class, whose somata normally lie within $20-100 \mu \mathrm{m}$ of the muscle, can receive retrograde signals and a high concentration of diffusible factors, but their somata have little or no interaction with extracellular matrix or membrane surface molecules.

We scored the incidence of GABA-IR, glycine-IR, and glutamate-IR cells for all three classes of neurons and found that both axon-muscle and soma-axon-muscle groups contain significantly fewer noncholinergic neurons than the no-contact group but are not significantly different from each other (Fig. $4 B$ ). This result implies that physical interaction between the neuronal soma and muscle cell body causes reduction of expression of noncholinergic transmitters. The data also suggest that retrograde signals propagated through axons play the key role in modulating transmitter expression. However, our results do not rule out the possibility that extracellular matrix molecules act directly on axons and activate pathways that lead to changes in transmitter expression in neuronal cell bodies (Hall and Sanes, 1993; Mantych and Ferreira, 2001).

To investigate whether a high concentration of diffusible factors contributes to the suppression of noncholinergic transmitter expression, we divided the no-contact group according to the distance from the neuronal soma to the nearest muscle cell. The concentration of diffusible factors that reaches neurons is expected to decline with distance from the muscle. However, no difference in the number of GABA-IR, glycine-IR, and glutamate-IR neurons was observed in neurons located $0-50$, $50-150,150-250$, and $>250 \mu \mathrm{m}$ from muscle cells (Fig. 4C). To further address the issue of diffusible factors, we cultured neurons in medium conditioned by muscle tissue for $48 \mathrm{~h}$, to parallel the time of neuronal exposure in neuron-muscle coculture. Muscle cells dissected from six embryos cover $70-80 \%$ of the dish. Trophic factors can be stable and remain active in culture medium for periods up to 1 week (Barnett et al., 1990). Thus, it was expected that the conditioned medium would provide relatively abundant diffusible factors released by the muscle. However, no changes were observed in the number of GABA-IR, glycine-IR, and glutamate-IR neurons, when the conditioned medium condition was compared with the control condition in which neurons were cultured in medium without exposure to muscle cells (Fig. 4D). Since the release of muscle-derived diffusible factors can also be dependent on muscle activity, in another set of experiments both neurons and muscle cells from six embryos were
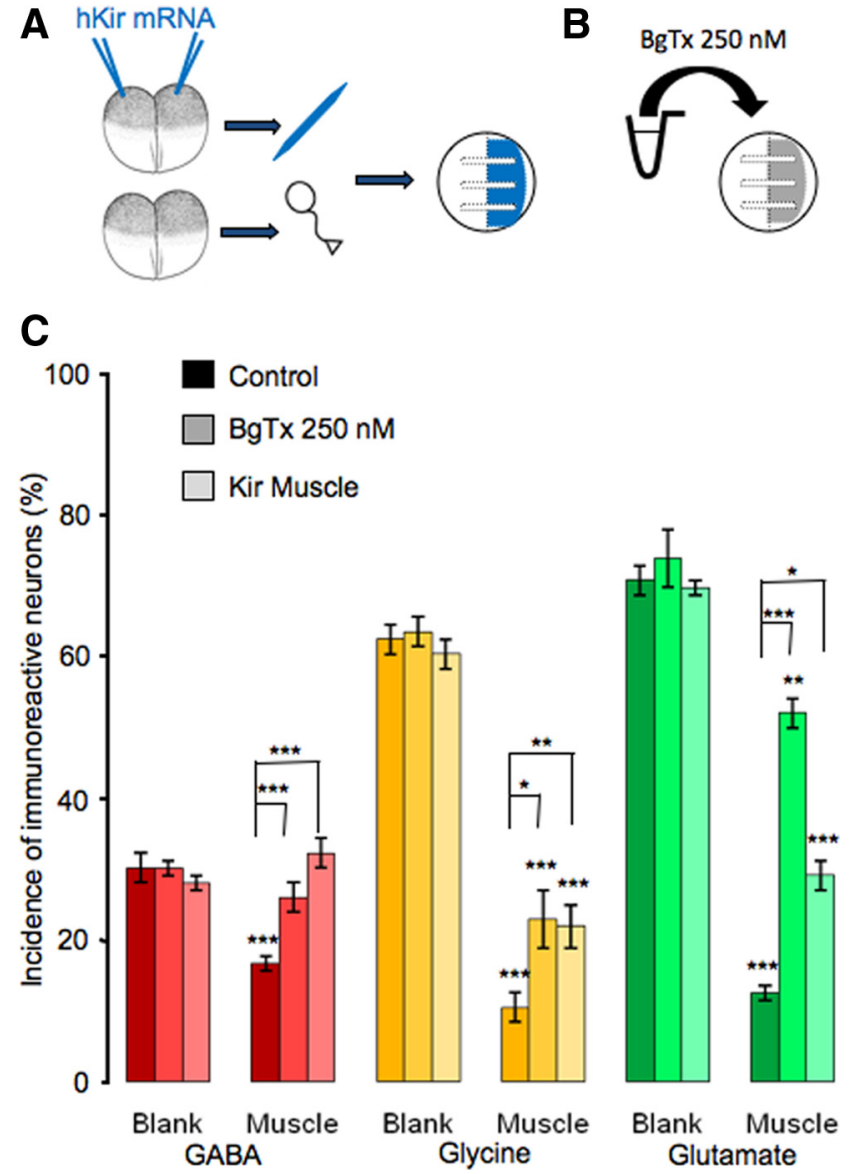

Figure 5. Rescue of noncholinergic neurotransmitter expression by blockade of muscle activity. $\boldsymbol{A}$, Diagram illustrating hKir mRNA injection and neuron-muscle coculture with silenced muscle and wild type neurons. $\boldsymbol{B}$, Diagram showing method of pharmacological blockade of muscle activity by $\alpha$-bungarotoxin. C, Complete rescue of GABA expression and partial rescue of glycine and glutamate expression are achieved by either BgTx incubation or Kir expression. Lower asterisks indicate a significant difference between the bar beneath and its counterpart on the blank side. Upper asterisks indicate a significant difference between the bars ( $n>5$ cultures per condition per transmitter, $>100$ neurons per culture; ${ }^{*} p<0.05,{ }^{* *} p<0.01,{ }^{* * *} p<$ $0.001)$. Error bars indicate SEM. The Kruskal-Wallis test and Conover post hoc test were used to determine statistical significance.

cocultured for $48 \mathrm{~h}$ before harvesting conditioned medium. Still no change in noncholinergic transmitter expression was observed (Fig. 4D). These findings suggest that diffusible factors play little role in suppressing noncholinergic transmitter expression, although the action of such factors over distances of a few micrometers or at very high concentrations is not excluded. Together, axon-muscle contact alone appears to be sufficient to cause significant suppression of noncholinergic transmitters on the muscle side of these cultures, and muscle-derived, retrogradely transported signals are likely to be the principal players in this suppression.

\section{Blocking muscle electrical activity rescues noncholinergic neurotransmitter expression}

To determine whether electrical activity in muscle cells is necessary to suppress noncholinergic neurotransmitter expression, we used two separate approaches to block muscle activity chronically. We first incubated cultures in $250 \mathrm{~nm} \mathrm{BgTx}$ to block postsynaptic activity mediated by acetylcholine receptors (Berg and Hall, 1975). In parallel experiments we heterologously expressed human Kir2.1 channels in embryos from which the 


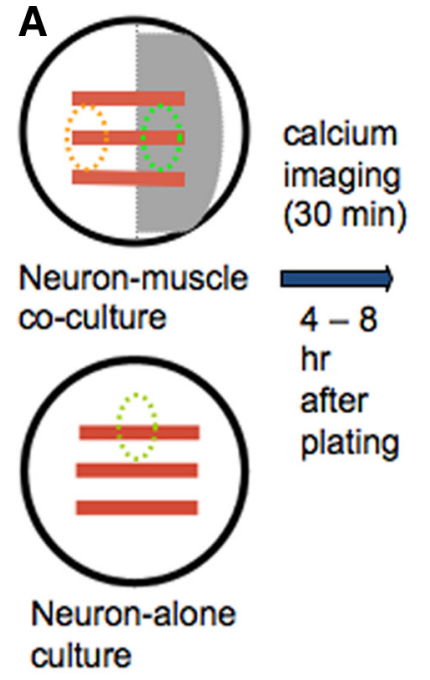

B
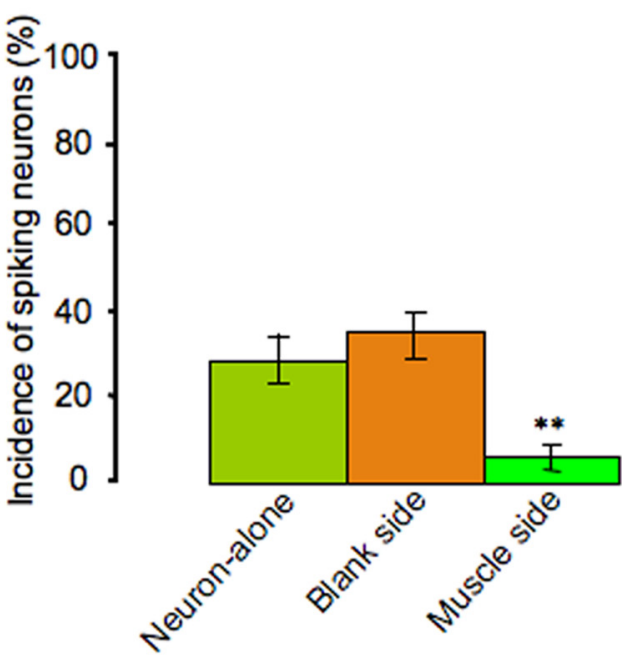

C

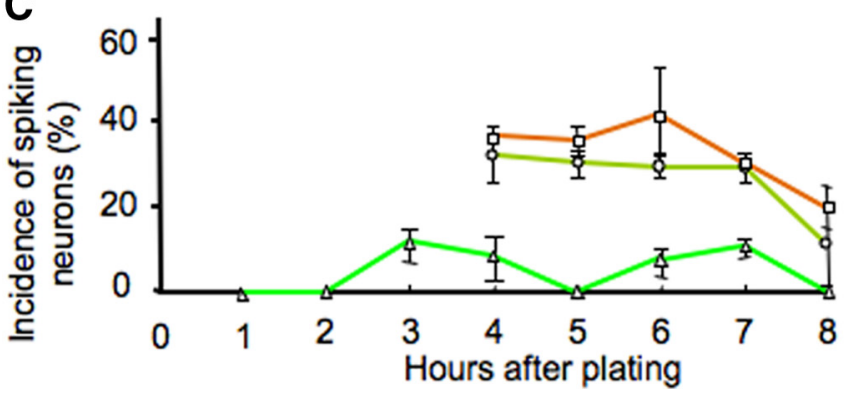

Figure 6. Muscle cells suppress early neuronal spontaneous calcium spike activity. $\boldsymbol{A}$, Diagram showing areas of interest in which neurons were imaged. $\boldsymbol{B}$, Neurons grown on muscle cells exhibit a significantly lower incidence of spiking at $4-8 \mathrm{~h}$ in vitro ( $n=10$ cultures, $>50$ neurons per condition; ${ }^{* *} p<0.01$ ). C, Neurons grown on muscle cells exhibit reduced incidence of spiking during each hour after plating ( $n>10$ cultures, $>50$ neurons per hour). A higher percentage of neurons exhibit calcium spikes from 4 to $8 \mathrm{~h}$ on the blank side of cocultures and in neuron-alone cultures. Imaging at earlier times was not performed with the two control groups due to lack of morphological distinction between neurons before axon outgrowth and the presence of other cell types. Error bars indicate SEM. The Kruskal-Wallis test and Conover post hoc test were used to determine statistical significance.

muscle cells were dissociated, to hyperpolarize their membrane potential (Borodinsky et al., 2004) (Fig. 5A,B). Neither of these two methods led to a change in noncholinergic transmitter expression in the neurons on the blank side (Fig. $5 C$ ), making it unlikely that the changes in transmitter expression in neurons on the muscle side are due to blockade of neuronal $\mathrm{nACh}$ receptors alone. On the muscle side, the percentage of GABA-IR-positive neurons was fully restored to the level on the blank side when the muscle was silenced by either BgTx or Kir expression. Significant although partial rescue of glycine-IR and glutamate-IR neurons was also achieved by both of these two methods. The number of cholinergic neurons is not sufficient to account for the increase in noncholinergic transmitter expression on the basis of direct innervation. Accordingly the paracrine release of ACh or another factor from cholinergic neurons and/or muscle cells (Fu et al., 1998) may exert this effect. Moreover the effective molecules are not exclusively taken up at synapses, although they appear to be taken up by nerve terminals and signals are retrogradely transported. These results demonstrate that muscle activity plays an essential role in suppressing expression of noncholinergic transmitters. However, the different extents of rescue for GABA, glycine, and glutamate indicate that the expression of different transmitters is regulated differently by the muscle target.
Neuron-muscle coculture suppresses embryonic neuronal calcium spike activity

We next investigated the interplay between muscle-derived factors and spontaneous activity in transmitter specification. Spontaneous neuronal calcium spike activity is observed at early stages of development and plays an important role in transmitter specification both in vitro and in vivo $(\mathrm{Gu}$ and Spitzer, 1995; Borodinsky et al., 2004, Dulcis and Spitzer, 2008). Calcium spikes are produced by calcium-dependent action potentials generated by voltage-dependent calcium channels and involve calciuminduced calcium release from calcium stores. They are usually first detected at the neural tube stage (stage 20) (Spitzer and Lamborghini, 1976; Holliday and Spitzer, 1990; Gu and Spitzer, 1993; Borodinsky et al., 2004), before synapse formation (Hayes and Roberts, 1973), and are generated for 10-12 h until the early larval stage $(\sim$ stage 32; Root et al., 2008). Calcium spike activity disappears as the action potential matures to a brief sodium-dependent impulse. Spontaneous activity is also observed during a limited time window in culture. It peaks from 5 to $10 \mathrm{~h}$ after plating and gradually decreases afterward (Gu et al., 1994; Gu and Spitzer, 1995; Chang and Spitzer, 2009).

To determine whether the presence of muscle cells affects this neuronal activity, we imaged intracellular calcium in neurons dissociated from neural tube stage Xenopus embryos and grown on the muscle side and the blank side of cocultures. Neurons growing in neuronal cultures were also imaged, as a further control (Fig. $6 \mathrm{~A}$ ). Images were acquired for a $30 \mathrm{~min}$ period, starting $4-8 \mathrm{~h}$ after plating. The incidence of spiking neurons was calculated by dividing the number of neurons exhibiting spike activity during an imaging period by the total number of neurons imaged. We observed a significantly lower percentage of spiking neurons on the muscle side of the culture dishes, when compared with those on the blank side or in neuronal cultures (5\% vs 32\% and 28\%) (Fig. 6B).

On the muscle side, neurons extend axons more rapidly and grow longer than on the blank side during the first $8 \mathrm{~h}$ after plating. To determine whether those neurons mature more rapidly and shift the period during which they generate spikes to earlier times in culture, we imaged them at $1-4 \mathrm{~h}$ after plating. However, only $6 \%$ of neurons grown on the muscle side generated spikes during this period (Fig. 6C). This result suggests that shifting of the spiking window toward earlier times is unlikely.

\section{Muscle cells abolish veratridine-induced neuronal activity-dependent neurotransmitter specification} Manipulations of neuronal calcium activity alter neurotransmitter expression both in cultures prepared from neural plate stage embryos and in vivo (Gu and Spitzer, 1995; Borodinsky et al., 2004). We repeated the in vitro experiment with neuronal cultures dissociated from neural tube stage embryos. Enhancing 
spiking activity by chronic incubation with $1 \mu \mathrm{M}$ veratridine, a drug blocking sodium channel inactivation, led to an increase in the number of neurons expressing the inhibitory transmitters GABA and glycine and a decrease in the number of neurons expressing the excitatory transmitter glutamate (Fig. $7 A, B$ ).

To investigate whether reduced spontaneous neuronal activity is responsible for muscle-dependent transmitter specification, we performed a rescue experiment by enhancing neuronal activity. Chronic treatment of cocultures with $1 \mu \mathrm{M}$ veratridine increased the incidence of spiking neurons on the blank side by $100 \%$ and restored the incidence of spiking neurons on the muscle side (Fig. 7C). Veratridine does not elicit calcium transients in muscle cells in nerve-muscle cocultures (Borodinsky and Spitzer, 2007). However, even with complete rescue of neuronal activity, there was no difference in the percentage of neurons expressing GABA, glycine, or glutamate on either side of the dish (Fig. 7D). This result suggests that suppression of neuronal activity by muscle contact does not account for muscledependent suppression of noncholinergic transmitter expression. In addition, the surprisingly unaltered transmitter expression on the blank side of the dish shows that diffusible muscle-derived factors are likely to be sufficient to eliminate veratridine-induced, activitydependent transmitter specification. Since presynaptic activity may be involved in stimulating the release of muscle-derived factors, the high level of neuronal activity induced by veratridine may cause muscle to dominate the regulation of transmitter expression.

\section{Blocking trk receptors does}

not rescue noncholinergic neurotransmitter expression

Muscle-derived members of the neurotrophin family are important for motor neuron development, including the induction and maintenance of ChAT expression (Chiu et al., 1994; Zurn et al., 1996) and establishment of mature electrophysiological properties of the NMJ (Funakoshi et al., 1995; Liou and Fu, 1997; Xie et al., 1997; Nick and Ribera, 2000). To determine whether neurotrophins play a role in suppressing noncholinergic transmitters, cultures were incubated with $10 \mathrm{~nm} \mathrm{K252a}$, a trk receptor blocker. K252a was applied $5 \mathrm{~h}$ after plating, to minimize prevention of neuronal differentiation. At this concentration no rescue of GABA, glycine, or glutamate expression was achieved (Fig. 8). At higher concentrations or when applied immediately before plating neurons, K252a reduced the number of morphologically distinguishable neurons while leaving the percentage of tubulinpositive cells unaltered; neurons displayed short neurites and abnormal hairy morphology both on cell bodies and along axons. Although morphological differentiation was clearly abnormal, concentrations of K252a up to $10 \mu \mathrm{M}$ were equally ineffective in rescuing expression of the three noncholinergic transmitters and no concentration-dependent effect of K252a on transmitter expression was observed (data not shown).

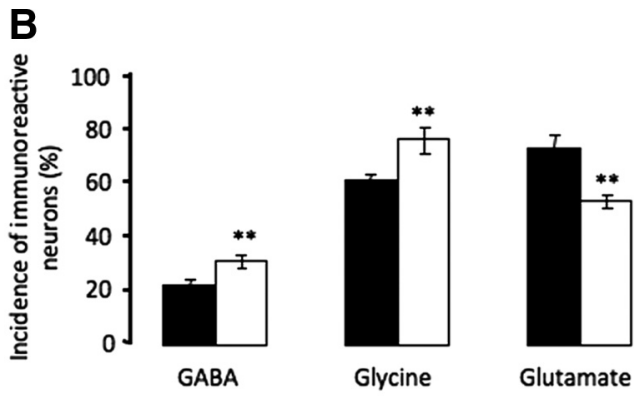

D

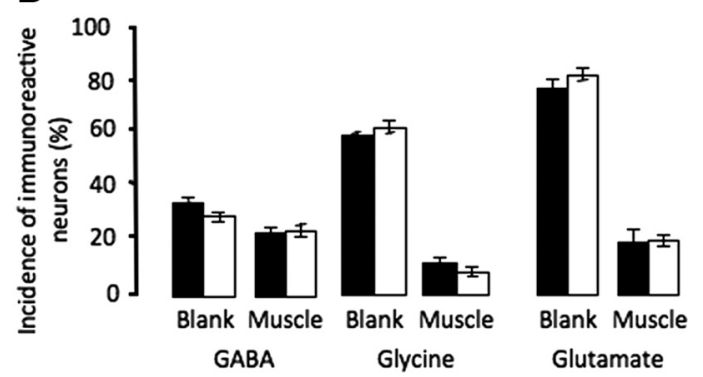




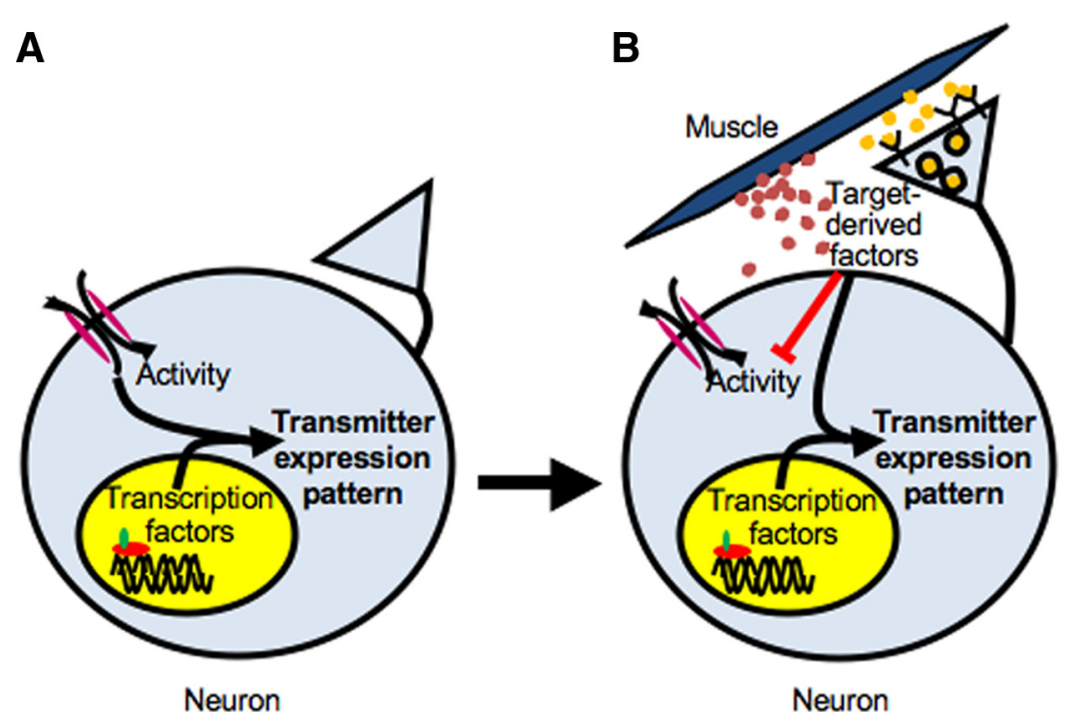

Figure 9. Model of neurotransmitter specification by transcription factors, activity, and target-derived factors. $\boldsymbol{A}$, Early in development, when neurons have not yet established contact with their targets, spontaneous calcium spike activity and transcription factors work together to specify their transmitter expression profile. $\boldsymbol{B}$, After neurons contact their targets, target-derived factors, including both retrograde factors (yellow dots) and diffusible factors (red dots), suppress expression of noncholinergic transmitters, block spontaneous calcium activity, and disrupt evoked activity-dependent transmitter specification. These factors work in combination with transcription factors to further refine and lock in the transmitter expression phenotype.

cocultured neurons, most likely via diffusible factors. These results demonstrate multilevel, muscle-dependent regulation of transmitter specification.

\section{Muscle cell-dependent neurotransmitter specification}

Previous studies demonstrated that muscle cells are critical to support neuronal survival and maintain ChAT expression in adult motor neurons (Lams et al., 1988; Kou et al., 1995). However, the initial expression of ChAT in motor neurons is thought to be target-independent, since rat thoracic spinal motor neurons express ChAT before they can be retrogradely labeled through their targets (Phelps et al., 1991). In Xenopus laevis, however, the onset of ChAT expression in spinal motor neurons is first detected at the tailbud stage (stage 30 ) by immunocytochemistry (Root et al., 2008), almost $12 \mathrm{~h}$ after axons start to contact developing muscle cells at the neural tube stage (stage 21) (Kullberg et al., 1977). Despite apparent differences between rat and Xenopus in the order of onset of ChAT expression and establishment of muscle contact, we found that the number of neurons expressing ChAT transcripts is not altered by contact with muscle cells. These results suggest that ChAT expression in embryonic Xenopus spinal neurons is indeed independent of muscle-derived factors.

Muscle-derived signals appear to have a profound effect on noncholinergic transmitter expression. In vivo, Xenopus spinal neurons start to express GABA and glutamate at neural plate stages, well before synapse formation (Root et al., 2008). Accordingly, when Xenopus neurons are dissociated and subsequently cultured from stage 20 embryos, it is expected that GABA and glutamate are present while glycine is absent. After $1 \mathrm{~d}$ in culture, expression of all three noncholinergic transmitters is much higher in neurons grown free of muscle contact, when compared with those grown on muscle cells. Thus, nerve-muscle contact seems to be effective both in reducing preexisting noncholinergic transmitter (GABA and glutamate) expression and in preventing neurons from acquiring a new noncholinergic transmitter (glycine). The expression of the intrinsic molecular markers HNK-1 and Islet-1 does not differ between the two groups of neurons, suggesting that muscle-dependent transmitter specification is independent of cell death.

In vivo, GABA and glutamate expression peaks at the neural tube stage, declining thereafter, and both transmitters are expressed in neurons that are positive for lim-3, a motor neuron transcription factor (Root et al., 2008). Since the reduction of GABA and glutamate expression occurs at the time when motor neurons start to contact muscle cells, muscle-derived retrograde signals may contribute to suppression of ectopic expression of GABA and glutamate in motor neurons in vivo. In addition, early tailbud stage (stage 25) neurons that are positive for lim-3 never exhibit glycine-IR. Consequently, neuron-muscle contact may also help to prevent motor neurons from acquiring the wrong transmitter in vivo.

\section{Role of muscle cell activity and candidate molecules}

Many target-derived factors are released in an activity-dependent fashion (Xie et al., 1997). Postsynaptic excitability is critical in forming functional synapses and in synapse maturation; synaptic activity often modulates synaptic efficiency, both at early developmental stages and in adults. Electrical stimulation of the rat sciatic nerve leads to increased levels of NT4 mRNA in both the soleus and gastrocnemius muscles and blocking postsynaptic activity blocks factor release and thus abolishes factor-dependent regulation of development (Funakoshi et al., 1995; Nick and Ribera, 2000). We find that blocking muscle activity suppresses reductions in expression of noncholinergic transmitters. The rescue is complete for GABA and partial for glutamate and glycine. This suggests that multiple factors or mechanisms are responsible for target-dependent regulation and that different transmitters are regulated in different ways. Because BgTx blocks nicotinic acetylcholine receptors that mediate transmission at the NMJ and rescues expression of noncholinergic transmitters on the muscle side of cocultures, presynaptic release of acetylcholine may lead to suppression of noncholinergic transmitter expression.

Since the lattice culture and conditioned medium experiments argue for the role of retrogradely transported trophic factors, it will be useful to identify the target-derived factors that are involved in regulating the changes in transmitter specification we have observed. Several neurotrophins have been shown to play important roles in muscle-dependent motor neuron differentiation. BDNF can rescue ChAT expression in adult mammalian hypoglossal neurons following transection and induce ChAT expression in cultured embryonic mouse neurons (Zurn et al., 1996; Liou and Fu, 1997). Both muscle-derived NT-3 and NT-4 regulate presynaptic electrophysiological properties in an activity-dependent manner (Funakoshi et al., 1995; Nick and Ribera, 2000). Although chronic blockade of trk receptors by K252a failed to rescue expression of the noncholinergic transmitters, we cannot exclude the possibility that different members of the neurotrophin family act differently or have opposing effects, in which case blocking them all could mask changes elicited by each one. Knocking down individual receptors would provide a more definitive test.

Many other factors also play important roles in motor neuron development (Zurn et al., 1996; Dryer et al., 2003). A number of 
target-derived factors, including CNTF, LIF, and GDNF have been proposed as potential candidates regulating the switch of rat sympathetic neurons from a noradrenergic to a cholinergic phenotype during development (Francis and Landis, 1999; Zweifel et al., 2005). BMP-9 is effective in inducing and maintaining the neuronal cholinergic phenotype in the CNS (López-Coviella et al., 2000). Pharmacological blockade and targeted gene knock-down or knock-out of specific receptors may be expected to identify muscle-derived factors responsible for transmitter specification in neuronal development.

\section{Muscle cell inhibition of early neuronal calcium spike activity} Spontaneous calcium-dependent spikes are characteristic of the early development of Xenopus spinal neurons, both in vivo and in vitro. During the first $8 \mathrm{~h}$ after plating, calcium spikes are rare in neurons grown on muscle although they are relatively common in neurons free of muscle contact. This result indicates that muscle cells prevent neurons from generating spontaneous spikes. It remains to be determined whether this result is due to the action of retrogradely transported signals or a high concentration of diffusible factors. Because veratridine not only rescues but also enhances spike incidence on the muscle side to the same extent as the blank side, calcium channels are not functionally impaired; however, the spike trigger mechanism may be suppressed. Spontaneous calcium spikes disappear as synapses mature in vivo, but this also happens in dissociated cell cultures in the absence of cell-cell interactions (Gu and Spitzer, 1995). It is not yet clear whether termination of this spontaneous activity is due to genetic programs or target-derived factors or to both.

\section{Muscle cells override activity-dependent transmitter specification}

Modulation of spontaneous calcium spike activity respecifies transmitter expression in a homeostatic manner. Altering this activity pharmacologically or by ion channel overexpression results in changes in transmitter expression that tend to counterbalance the effects of activity manipulation. Like spontaneous calcium spike activity, activity-dependent transmitter specification occurs only during a brief time window during development (Borodinsky et al., 2004; Root et al., 2008) before synapse formation.

In neuron-muscle cocultures, however, even when neuronal activity is altered, no change in transmitter expression takes place. More remarkably, abolition of activity-dependent transmitter specification occurs with or without physical contact between neurons and muscle cells, as long as they are placed in the same dish, arguing for regulation by diffusible factors. Because diffusible factors seem to play little or no role in reducing noncholinergic transmitter expression, it appears that different factors and target-dependent mechanisms are responsible for these two aspects of neuronal differentiation. In addition, because target-derived factors can be released in an activity-dependent fashion, it is likely that veratridine enhances neuronal activity and in turn enhances postsynaptic muscle activity, which leads to increased release of trophic factors that abolish activitydependent transmitter specification. These results suggest that muscle provides factors that prevent neurons from resuming activity-dependent transmitter specification even if calciumdependent neuronal activity were to return at a later time.

\section{Model for target-dependent regulation of neurotransmitter expression}

We propose a model in which different combinations of intrinsic genetic coding, neuronal activity and target-derived factors at different stages define the pattern of neurotransmitter expression. The pattern is initially specified genetically through the activity of transcription factors and modulated by environmental influences through changes in spontaneous calcium-dependent activity (Fig. 9A). Once neurons have made contact with their targets, the influence of target-derived factors, including both retrogradely transported signals and diffusible factors, overrides that of early activity. Targets block spontaneous calcium spike activity and further modulate the action of transcription factors to refine the neuronal transmitter expression phenotype (Fig. $9 B$ ). Thus, several mechanisms are used to ensure that the correct transmitter phenotype is established and maintained throughout development.

\section{References}

Barnett J, Baecker P, Routledge-Ward C, Bursztyn-Pettegrew H, Chow J, Nguyen B, Bach C, Chan H, Tuszynski MH, Yoshida K (1990) Human beta nerve growth factor obtained from a baculovirus expression system has potent in vitro and in vivo neurotrophic activity. Exp Neurol 110:11-24.

Berg DK, Hall ZW (1975) Increased extrajunctional acetylcholine sensitivity produced by chronic acetylcholine sensitivity produced by chronic post-synaptic neuromuscular blockade. J Physiol 244:659-676.

Borodinsky LA, Spitzer NC (2007) Activity-dependent neurotransmitterreceptor matching at the neuromuscular junction. Proc Natl Acad Sci U S A 104:335-340.

Borodinsky LA, Root CM, Cronin JA, Sann SB, Gu X, Spitzer NC (2004) Activity-dependent homeostatic specification of transmitter expression in embryonic neurons. Nature 420:523-530.

Brosenitsch TA, Katz DM (2002) Expression of Phox2 transcription factors and induction of the dopaminergic phenotype in primary sensory neurons. Mol Cell Neurosci 20:447-457.

Chang LW, Spitzer NC (2009) Spontaneous calcium spike activity in embryonic spinal neurons is regulated by developmental expression of the $\mathrm{Na}^{+}, \mathrm{K}^{+}$-ATPase $\beta 3$ subunit. J Neurosci 29:7877-7885.

Chiu AY, Chen EW, Loera S (1994) Distinct neurotrophic responses of axotomized motor neurons to BDNF and CNTF in adult rats. Neuroreport 5:693-696.

Dryer SE, Lhuillier L, Cameron JS, Martin-Caraballo M (2003) Expression of KCa channels in identified populations of developing vertebrate neurons: role of neurotrophic factors and activity. J Physiol Paris 97:49-58.

Dulcis D, Spitzer NC (2008) Illumination controls differentiation of dopamine neurons regulating behavior. Nature 456:195-201.

Fernandes KJ, Kobayashi NR, Jasmin BJ, Tetzlaff W (1998) Acetylcholinesterase gene expression in axotomized rat facial motoneurons is differentially regulated by neurotrophins: correlation with trkB and trkC mRNA levels and isoforms. J Neurosci 18:9936-9947.

Francis NJ, Landis SC (1999) Cellular and molecular determinants of sympathetic neuron development. Annu Rev Neurosci 22:541-566.

Fu WM, Liou HC, Chen YH, Wang SM (1998) Release of acetylcholine from embryonic myocytes in Xenopus cell cultures. J Physiol 509:497-506.

Funakoshi H, Belluardo N, Arenas E, Yamamoto Y, Casabona A, Persson H, Ibáñez CF (1995) Muscle-derived neurotrophin-4 as an activity-dependent trophic signal for adult motor neurons. Science 268:1495-1499.

Furshpan EJ, MacLeish PR, O'Lague PH, Potter DD (1976) Chemical transmission between rat sympathetic neurons and cardiac myocytes developing in microcultures: evidence for cholinergic, adrenergic, and dual function neurons. Proc Natl Acad Sci U S A 73:4225-4229.

Gu X, Spitzer NC (1993) Low-threshold $\mathrm{Ca}^{2+}$ current and its role in spontaneous elevations of intracellular $\mathrm{Ca}^{2+}$ in developing Xenopus neurons. J Neurosci 13:4936-4948.

Gu X, Spitzer NC (1995) Distinct aspects of neuronal differentiation encoded by frequency of spontaneous $\mathrm{Ca}^{2+}$ transients. Nature 375:784-787.

Gu X, Olson EC, Spitzer NC (1994) Spontaneous neuronal calcium spikes and waves during early differentiation. J Neurosci 14:6325-6335.

Hall ZW, Sanes JR (1993) Synaptic structure and development: the neuromuscular junction. Cell [Suppl] 72:99-121.

Hartenstein V (1989) Early neurogenesis in Xenopus: the spatio-temporal pattern of proliferation and cell lineages in the embryonic spinal cord. Neuron 3:399-411. 
Hayes BP, Roberts A (1973) Synaptic junction development in the spinal cord of an amphibian embryo: an electron microscope study. Z Zellforsch Mikrosk Anat 137:251-269.

Henderson LP, Smith MA, Spitzer NC (1984) The absence of calcium blocks impulse-evoked release of acetylcholine but not de novo formation of functional neuromuscular synaptic contacts in culture. J Neurosci 4:3140-3150.

Holliday J, Spitzer NC (1990) Spontaneous calcium influx and its roles in differentiation of spinal neurons in culture. Dev Biol 141:13-23.

Holliday J, Spitzer NC (1993) Calcium regulates neuronal differentiation both directly and via co-cultured myocytes. J Neurobiol 24:506-514.

Jiang JX, Choi RC, Siow NL, Lee HH, Wan DC, Tsim KW (2003) Muscle induces neuronal expression of acetylcholinesterase in neuron-muscle co-culture. J Biol Chem 278:45435-45444.

Joshi K, Lee S, Lee B, Lee JW, Lee SK (2009) LMO4 Controls the balance between excitatory and inhibitory spinal V2 interneurons. Neuron 61:839-851.

Kou SY, Chiu AY, Patterson PH (1995) Differential regulation of motor neuron survival and choline acetyltransferase expression following axotomy. J Neurobiol 27:561-572.

Kullberg RW, Lentz TL, Cohen MW (1977) Development of the myotomal neuromuscular junction in Xenopus laevis: an electrophysiological and fine-structural study. Dev Biol 60:101-129.

Lams BE, Isacson O, Sofroniew MV (1988) Loss of transmitter-associated enzyme staining following axotomy does not indicate death of brain-stem cholinergic neurons. Brain Res 475:401-406.

Landis SC, Keefe D (1983) Evidence for neurotransmitter plasticity in vivo: developmental changes in properties of cholinergic sympathetic neurons. Dev Biol 98:349-372.

Lee SK, Pfaff SL (2001) Transcriptional networks regulating neuronal identity in the developing spinal cord. Nat Neurosci 4:1183-1191.

Liou JC, Fu WM (1997) Regulation of quantal secretion from developing motoneurons by postsynaptic activity-dependent release of NT-3. J Neurosci 17:2459-2468.

Liu X, Popescu IR, Denisova JV, Neve RL, Corriveau RA, Belousov AB (2008) Regulation of cholinergic phenotype in developing neurons. J Neurophysiol 99:2443-2455.

López-Coviella I, Berse B, Krauss R, Thies RS, Blusztajn JK (2000) Induction and maintenance of the neuronal cholinergic phenotype in the central nervous system by BMP-9. Science 289:313-316.

Mantych KB, Ferreira A (2001) Agrin differentially regulates the rates of axonal and dendritic elongation in cultured hippocampal neurons. J Neurosci 21:6802-6809.
Nawa H, Patterson PH (1990) Separation and partial characterization of neuropeptide-inducing factors in heart cell conditioned medium. Neuron 4:169-277.

Nick TA, Ribera AB (2000) Synaptic activity modulates presynaptic excitability. Nat Neurosci 3:142-149.

Pfaff SL, Mendelsohn M, Stewart CL, Edlund T, Jessell TM (1996) Requirement for LIM homeobox gene Isl1 in motor neuron generation reveals a motor neuron-dependent step in interneuron differentiation. Cell 84:309-320.

Phelps PE, Barber RP, Vaughn JE (1991) Embryonic development of choline acetyltransferase in thoracic spinal motor neurons: somatic and autonomic neurons may be derived from a common cellular group. J Comp Neurol 307:77-86.

Ribera AB, Spitzer NC (1989) A critical period of transcription required for differentiation of the action potential of spinal neurons. Neuron 2:1055-1062.

Root CM, Velázquez-Ulloa NA, Monsalve GC, Minakova E, Spitzer NC (2008) Embryonically expressed GABA and glutamate drive electrical activity regulating neurotransmitter specification. J Neurosci 28:47774784.

Somasekhar T, Nordlander RH (1997) Selective early innervation of a subset of epidermal cells in Xenopus may be mediated by chondroitin sulfate proteoglycans. Brain Res Dev Brain Res 99:208-215.

Spitzer NC (2006) Electrical activity in early neuronal development. Nature 444:707-712.

Spitzer NC, Lamborghini JE (1976) The development of the action potential mechanism of amphibian neurons isolated in cell culture. Proc Natl Acad Sci U S A 73:1641-1645.

Tanabe Y, William C, Jessell TM (1998) Specification of motor neuron identity by the MNR2 homeodomain protein. Cell 95:67-80.

Timmusk T, Belluardo N, Metsis M, Persson H (1993) Widespread and developmentally regulated expression of neurotrophin-4 mRNA in rat brain and peripheral tissues. Eur J Neurosci 5:605-613.

Xie K, Wang T, Olafsson P, Mizuno K, Lu B (1997) Activiy-dependent expression of NT-3 in muscle cells in culture: implications in development of neuromuscular junctions. J Neurosci 17:2947-2958.

Zurn AD, Winkel L, Menoud A, Djabali K, Aebischer P (1996) Combined effects of GDNF, BDNF and CNTF on motoneuron differentiation in vitro. J Neurosci Res 44:133-141.

Zweifel LS, Kuruvilla R, Ginty DD (2005) Functions and mechanisms of retrograde neurotrophin signaling. Nat Rev Neurosci 6:615-625. 